\title{
Taking a step toward implementation of Global Leadership Initiative on Malnutrition (GLIM) criteria in geriatric rehabilitation
}

\author{
Dolores Sanchez-Rodriguez ${ }^{1,2,3,4} \cdot$ Ester Marco $^{4,5,6} \cdot$ Delky Meza-Valderrama ${ }^{3,4,5}$. Vanesa Dávalos-Yerovi,4,5 \\ Esther Duarte ${ }^{4,5,7}$
}

Published online: 29 April 2020

(c) European Geriatric Medicine Society 2020

The manuscript "Nutritional status and functionality in geriatric rehabilitation patients: a systematic review and meta-analysis" [1] by Wojzischke et al. provides updated evidence on an important topic, lists the tools and criteria used to identify malnutrition, determines its prevalence, and assesses the relationship between malnutrition and functional outcomes in geriatric rehabilitation. We consider the management of nutritional disorders to be a cornerstone in geriatric rehabilitation units as we all seek to administer comprehensive geriatric assessment and intervention programs to improve patients' functional status after a deconditioning, eventually reversible process. To further contextualize the findings of this timely review, the overall objective of this editorial is to raise awareness about malnutrition and nutrition-related diseases in geriatric care. The aim is to help specialists in charge of these units to improve quality of diagnosis and nutritional care in geriatric rehabilitation.

Dolores Sanchez-Rodriguez

dolores.sanchez@uliege.be; 97662@parcdesalutmar.cat

1 WHO Collaborating Centre for Public Health Aspects of Musculo-Skeletal Health and Ageing, Division of Public Health, Epidemiology and Health Economics, University of Liège, CHU, Sart Tilman. Quartier Hôpital. Avenue Hippocrate 13 (Bât. B23), 4000 Liège, Belgium

2 Geriatrics Department, Hospital Del Mar Medical Research Institute (IMIM), Barcelona, Spain

3 Facultat de Ciències de la Salut i de la Vida, Universitat Pompeu Fabra, Barcelona, Spain

4 Rehabilitation Research Group, Hospital Del Mar Medical Research Institute (IMIM), Universitat Pompeu Fabra, Barcelona, Spain

5 Physical Medicine and Rehabilitation Department, Hospital Del Mar Medical Research Institute (IMIM), Barcelona, Spain

6 Universitat International de Catalunya, Barcelona, Spain

7 Department of Medicine, Medicine School, Universitat Autònoma de Barcelona, Barcelona, Spain
In older adults, malnutrition is related to impaired functional status, falls [2], decreased probability of being discharged home [3], longer length of hospital stay [4], and higher risk of all-cause mortality [5], whether community-dwelling [6], hospitalized [7], or post-discharge [3]. Unfortunately, proper identification and management of a patient with malnutrition may depend on the diagnostic tool administered $[8,5]$. This presents an important challenge for clinicians.

Aware of the adverse consequences of malnutrition in older people and the promising outcomes that are possible with good nutritional care, the European Geriatric Medicine Society (EuGMS) has supported several initiatives aiming to better diagnose malnutrition, i.e., the European Society for Clinical Nutrition and Metabolism (ESPEN) consensus, which provided the first international definition of malnutrition [9], followed by the ESPEN guidelines on definition and terminology of clinical nutrition [10] and the Global Leadership Initiative on Malnutrition (GLIM) criteria [11]. Moreover, initiatives such as the PROT-Age recommendations [12] and the ESPEN guidelines on nutrition and hydration in Geriatrics [13] have been developed to update therapeutic approaches.

Malnutrition would be expected to be more prevalent and produce more severe negative outcomes in those older patients who have recently presented an acute catabolic process and whose metabolic needs are not well balanced [14], such as patients admitted in geriatric rehabilitation units [15]; likewise, an adequate diagnosis and management of malnutrition would be expected to have greater positive impact in these patients [13].

As these units have emerged to meet the needs of different populations and healthcare systems [16], they may differ by country but do share some common characteristics. Among the shared concerns are the disabling (hip fracture, acute stroke) or non-disabling processes (e.g., acute medical conditions) that lead to a loss of physical performance and 
independence, along with the need to optimize results within a limited treatment period. The length of the physical therapy program and length of admission in the unit are usually determined by the timeframe during which a deconditioning process remains sensitive to physical therapy interventions, by the achievement of goals, and by conditions of reimbursement from the different health systems. Last but not least, the achievement of a level of physical independence that allows the patient to return home and avoid institutionalization is equally or more important than the use of healthcare resources. A wide variety of standards of effectiveness have been proposed for geriatric rehabilitation [17]; however, most settings use their own pragmatic thresholds in the daily clinical decision-making process.

Apart from variations determined by the local setting and level of therapeutic intensity of the rehabilitation program, approaches differ depending on the relative weight of the rehabilitation therapy vs. patients' other needs. All these considerations should help to establish the roles of specialists leading these settings, who frequently represent Geriatrics and/or Physical Medicine and Rehabilitation Medicine and are supported by interdisciplinary teams of nurses, physiotherapists, social workers, and others who are crucial for improving patient's health during and after a hospital stay. Sharing knowledge and raising awareness about malnutrition and nutrition-related diseases between these medical specialties is well aligned with both research and clinical points of view [18].

However, high-quality evidence on the current assessment of malnutrition in light of the latest findings in geriatric rehabilitation has been lacking, and the meta-analysis by Wojzischke et al. helps to bridge this gap. They report a pooled prevalence of malnutrition of $13 \%(5-20 \%)$ and risk of malnutrition of 47\% (40-54\%) using the Mini-Nutritional Assessment (MNA) [19, 20] and a prevalence of malnutrition that ranged from 6 to $88 \%$ according to other assessment methods. These heterogeneous results confirm that a large percentage of patients remain either undiagnosed and undertreated or overdiagnosed, depending on the assessment tool used [15].

The ESPEN consensus [9] and the GLIM criteria [11] formulated several PICO questions during their respective development processes. One of these was "Which are the individual criteria that better capture the state of malnutrition?" [21]. Wojzischke et al. compiled the list of individual variables that constitute the ESPEN consensus and GLIM criteria as an outline to present the findings of their systematic review and meta-analysis: screening of malnutrition by validated screening tools, such as MNA or MNA-Short Form (MNA-SF) and diagnosis by low body mass index $(\mathrm{BMI})<20 \mathrm{~kg} / \mathrm{m}^{2}$, reduced muscle mass, and reduced nutritional intake. The meta-analysis concluded that 2 of the 3 individual criteria of the ESPEN consensus and 5 of the 6
GLIM criteria were used in the studies identified by their literature search; however, these were applied idiosyncratically, rather than as indicated by a consensus definition. Similar findings have been reported by an international survey on malnutrition assessment and diagnostic methods in clinical practice across European countries [22]. This wide use might indicate acknowledgement by a large percentage of the scientific and clinical community of the criteria that best capture the state of malnutrition, which can be considered promising for an eventual implementation of the GLIM criteria worldwide.

Although low BMI was widely used in the studies identified by the search strategy, this factor alone was not a good indicator of malnutrition, as a large percentage of malnourished patients according to MNA had normal BMI. Moreover, the meta-analysis did not show an association between BMI and physical function, which might indicate that evidence-based tools could be more accurate to diagnose malnutrition than a single phenotypic measurement. This observation is aligned with recent studies showing better performance of GLIM compared to simpler approaches using fewer criteria [23].

Furthermore, malnutrition according to the ESPEN consensus and GLIM criteria has been shown to be a strong predictor (fourfold increased risk) of sarcopenia onset during 5-year follow-up in older people living in the community [24]. However, despite this clear association, Wojzischke et al. [1] reveal a lack of accurate data regarding decreased muscle strength, suggesting that the assessment of muscle mass remains an ongoing challenge in the evaluation of body composition. Although a majority of the reviewed studies did not show data on prevalence of sarcopenia, two studies found high prevalence rates ( $40 \%$ and $76 \%$ ), which are consistent with the mean prevalence (50\%) reported in a review about sarcopenia in older adults during post-acute care and rehabilitation for a special issue of this journal [27]. Notably, the definition of sarcopenia has been recently updated by the European Working Group on Sarcopenia in Older People (EWGSOP2) [25] and could yield different results [26].

Another interesting concept developed by Wojzischke et al. is the association of malnutrition with poor functional outcomes as measured by the Barthel Index (BI) and the Functional Independent Measure (FIM). The findings show that malnutrition has a negative impact on functional outcomes and that worse BI or FIM is associated with malnutrition and/or malnutrition risk. The results also reinforce the importance of systematic screening in clinical practice using a validated tool such as MNA or MNA-SF, as recommended by ESPEN guidelines [13]. A limitation of the systematic review was that the search strategy did not include outcome variables other than BI and FIM during the hospital stay; these measures might not yield adequate information about the dynamic results of a 
rehabilitation program. Functional indexes such as absolute functional gain (admission-to-discharge BI change), rehabilitation efficiency index (absolute functional gain over length of stay), relative functional gain (achieved percentage of potential gain), and Heinemann index, among others, are indicators of the results of the intervention, can be used as goals, and help to support the clinical decisionmaking process in geriatric rehabilitation [17].

Wojzischke et al. present the available evidence about a fascinating, cutting-edge research topic: the effectiveness of the new criteria for the diagnosis of malnutrition and their relationship with nutrition-related diseases. Studies about the capability of the new GLIM criteria to predict adverse health outcomes are still scarce [26] and could be timely. Moreover, further research about pragmatic approaches [27] suitable for bedside assessment will be helpful for the widespread implementation of the ESPEN definition. Despite ESPEN and EuGMS support of the GLIM criteria [28], and the interest that the criteria aroused among European geriatric societies, no European guidelines to date recommend their use [22]. The relative novelty of the GLIM criteria and their limited diffusion among clinicians, the pitfalls of measuring muscle mass [22, 29-31], the still frequent use of serum albumin levels as a measurement of nutritional status [36], and resistance to change in daily practice [32] remain the major limitations for the implementation of the new criteria in clinical practice in geriatric rehabilitation. The meta-analysis published in this issue could be a promising starting point for a line of research in malnutrition and nutrition-related diseases in geriatric rehabilitation designed to improve nutritional care in older people.

Acknowledgements The authors appreciate the editorial assistance and support provided by Elaine M. Lilly, Ph.D.

Author contributions DSR, DMV, and VDY wrote the manuscript; DSR, DMV, VDY did literature review; EM and ED corrected the manuscript. All co-authors read and approved the final version of the manuscript.

Funding English language editing of this manuscript was supported by a Support for Research Groups Grant (2017 SGR 112) from the Agency for Management of University and Research Grants, Generalitat de Catalunya.

\section{Compliance with ethical standards}

Conflict of interest On behalf of all authors, the corresponding author states that there is no conflict of interest.

Ethical approval This study does not contain any studies with human participants performed by any of the authors.

Informed consent For this type of study, formal consent is not required.

\section{References}

1. Bauer JMJ, Wojzischke J, van Wijngaarden J et al (2020) Nutritional status and functionality in geriatric rehabilitation patients: a systematic review and meta-analysis. Eur Geriatr Med. https:// doi.org/10.1007/s41999-020-00294-2

2. Adly NN, Abd-El-Gawad WM, Abou-Hashem RM (2019) Relationship between malnutrition and different fall risk assessment tools in a geriatric in-patient unit. Aging Clin Exp Res. https:// doi.org/10.1007/s40520-019-01309-0

3. Sánchez-Rodríguez D, Annweiler C, Ronquillo-Moreno $\mathrm{N}$ et al (2019) Prognostic value of the ESPEN consensus and guidelines for malnutrition: prediction of post-discharge clinical outcomes in older inpatients. Nutr Clin Pract 34:304-312. https://doi. org/10.1002/ncp. 10088

4. Sanz-París A, Gómez-Candela C, Martín-Palmero Á et al (2016) Application of the new ESPEN definition of malnutrition in geriatric diabetic patients during hospitalization: a multicentric study. Clin Nutr 35:1564-1567. https://doi.org/10.1016/j. clnu.2016.02.018

5. Sanchez-Rodriguez D, Locquet M, Reginster J-Y et al (2020) Mortality in malnourished older adults diagnosed by ESPEN and GLIM criteria in the SarcoPhAge study. J Cachexia Sarcopenia Muscle. https://doi.org/10.1002/jcsm.12574 (in press)

6. Sánchez-Rodríguez D, Marco E, Schott A et al (2019) Malnutrition according to ESPEN definition predicts long-term mortality in general older population: findings from the EPIDOS study-Toulouse cohort. Clin Nutr 38:2652-2658. https://doi.org/10.1016/j. clnu.2018.11.016

7. Sánchez-Rodríguez D, Marco E, Annweiler C et al (2017) Malnutrition in postacute geriatric care: basic ESPEN diagnosis and etiology based diagnoses analyzed by length of stay, in-hospital mortality, and functional rehabilitation indexes. Arch Gerontol Geriatr 73:169-176. https://doi.org/10.1016/j.archger.2017.07.010

8. Sánchez-Rodríguez D, Annweiler C, Ronquillo-Moreno $\mathrm{N}$ et al (2018) Clinical application of the basic definition of malnutrition proposed by the European Society for clinical nutrition and Metabolism (ESPEN): comparison with classical tools in geriatric care. Arch Gerontol Geriatr 76:210-214. https://doi.org/10.1016/j. archger.2018.03.007

9. Cederholm T, Bosaeus I, Barazzoni R et al (2015) Diagnostic criteria for malnutrition - an ESPEN consensus statement. Clin Nutr 34:335-340. https://doi.org/10.1016/j.clnu.2015.03.001

10. Cederholm T, Barazzoni R, Austin P et al (2017) ESPEN guidelines on definitions and terminology of clinical nutrition. Clin Nutr 36:49-64. https://doi.org/10.1016/j.clnu.2016.09.004

11. Yilmaz M, Atilla FD, Sahin F et al (2019) The effect of malnutrition on mortality in hospitalized patients with hematologic malignancy. Support Care Cancer. https://doi.org/10.1007/s0052 0-019-04952-5

12. Bauer J, Biolo G, Cederholm T et al (2013) Evidence-based recommendations for optimal dietary protein intake in older people: a position paper from the prot-age study group. J Am Med Dir Assoc 14:542-559. https://doi.org/10.1016/j.jamda.2013.05.021

13. Volkert D, Beck AM, Cederholm T et al (2019) ESPEN guideline on clinical nutrition and hydration in geriatrics. Clin Nutr 38:10-47. https://doi.org/10.1016/j.clnu.2018.05.024

14. Agarwal E, Ferguson M, Banks M et al (2013) Malnutrition and poor food intake are associated with prolonged hospital stay, frequent readmissions, and greater in-hospital mortality: results from the nutrition care day survey 2010. Clin Nutr 32:737-745. https:// doi.org/10.1016/j.clnu.2012.11.021

15. Marshall S, Young A, Bauer J et al (2016) Malnutrition in geriatric rehabilitation: prevalence, patient outcomes, and criterion validity of the scored patient-generated subjective global assessment and 
the mini nutritional assessment. J Acad Nutr Diet 116:785-794. https://doi.org/10.1016/j.jand.2015.06.013

16. Rubenstein LZ, Abrass IB, Kane RL (1981) Improved care for patients on a new geriatric evaluation unit. J Am Geriatr Soc 29:531-536

17. Sánchez-Rodríguez D, Miralles R, Muniesa JM et al (2015) Three measures of physical rehabilitation effectiveness in elderly patients: a prospective, longitudinal, comparative analysis. BMC Geriatr 15:142. https://doi.org/10.1186/s12877-015-0138-5

18. Meza-Valderrama D, Marco E, Duarte E (2020) Evaluación de la masa muscular en la práctica clínica en rehabilitación. Rehabilitación 54:1-2. https://doi.org/10.1016/j.rh.2020.01.001

19. Guigoz Y, Lauque S, Vellas BJ (2002) Identifying the elderly at risk for malnutrition. The mini nutritional assessment. Clin Geriatr Med 18:737-757

20. Kaiser MJ, Bauer JM, Ramsch C et al (2009) Validation of the mini nutritional assessment short-form (MNA-SF): a practical tool for identification of nutritional status. J Nutr Health Aging 13:782-788

21. Cederholm T, Jensen GL (2017) To create a consensus on malnutrition diagnostic criteria: a report from the global leadership initiative on malnutrition (GLIM) meeting at the ESPEN congress 2016. Clin Nutr 36:7-10. https://doi.org/10.1016/j. clnu.2016.12.001

22. Sanchez-Rodriguez D, Annweiler C, Marco E et al (2020) European Academy for medicine of ageing session participants' report on malnutrition assessment and diagnostic methods; an international survey. Clin Nutr ESPEN 35:75-80. https://doi. org/10.1016/j.clnesp.2019.11.007

23. Allard JP, Keller H, Gramlich L et al (2019) GLIM criteria has fair sensitivity and specificity for diagnosing malnutrition when using SGA as comparator. Clin Nutr. https://doi.org/10.1016/j. clnu.2019.12.004

24. Beaudart C, Sanchez-Rodriguez D, Locquet M et al (2019) Malnutrition as a strong predictor of the onset of sarcopenia. Nutrients 11:2883. https://doi.org/10.3390/nu11122883

25. Cruz-Jentoft AJ, Bahat G, Bauer J et al (2019) Sarcopenia: revised European consensus on definition and diagnosis. Age Ageing 48:601. https://doi.org/10.1093/ageing/afz046
26. Sanchez-Rodriguez D, Marco E, Cruz-Jentoft AJ (2020) Defining sarcopenia: some caveats and challenges. Curr Opin Clin Nutr Metab Care 23:127-132. https://doi.org/10.1097/MCO.00000 00000000621

27. Contreras B, Sánchez T, Ruiz V et al (2019) GLIM criteria using hand grip strength adequately predict six-month mortality in cancer inpatients. Nutrients 11:2043. https://doi.org/10.3390/nu110 92043

28. Piotrowicz K, Fähling K, Roubaud-Baudron C et al (2019) Highlights of the 14th international congress of the European geriatric medicine society. Eur Geriatr Med 10:995-998. https://doi. org/10.1007/s41999-019-00238-5

29. Buckinx F, Landi F, Cesari M et al (2018) Pitfalls in the measurement of muscle mass: a need for a reference standard. J Cachexia Sarcopenia Muscle 9:269-278. https://doi.org/10.1002/ jcsm. 12268

30. Beaudart C, McCloskey E, Bruyère $\mathrm{O}$ et al (2016) Sarcopenia in daily practice: assessment and management. BMC Geriatr 16:170. https://doi.org/10.1186/s12877-016-0349-4

31. Landi F, Camprubi-Robles M, Bear DEE et al (2018) Muscle loss: the new malnutrition challenge in clinical practice. Clin Nutr 38:2113-2120. https://doi.org/10.1016/j.clnu.2018.11.021

32. Sanchez-Rodriguez D, Annweiler C, Marco E, Hope S, Piotrowicz K, Surquin M, Ranhoff A, Van Den Noortgate N, EAMA Board Members XII seminar participants and respondents of the survey (2020) European academy for medicine of ageing seminar participants report on malnutrition assessment methods in geriatric healthcare settings in the era of the global leadership initiative on malnutrition (GLIM) Criteria and the European Society for Clinical Nu. Clin Nutr ESPEN 35:75-80

Publisher's Note Springer Nature remains neutral with regard to jurisdictional claims in published maps and institutional affiliations. 